\title{
Reflets
}

Revue d'intervention sociale et communautaire

\section{Actualité de la pauvreté : débats théoriques, défis pratiques}

Dahlia Namian, Sarah Boucher-Guèvremont et Martin Chartrand

Volume 25, numéro 1, printemps 2019

Actualité de la pauvreté : débats théoriques, défis pratiques

URI : https://id.erudit.org/iderudit/1064664ar

DOI : https://doi.org/10.7202/1064664ar

Aller au sommaire du numéro

Éditeur(s)

Reflets, Revue d'intervention sociale et communautaire

ISSN

1203-4576 (imprimé)

1712-8498 (numérique)

Découvrir la revue

Citer ce document

Namian, D., Boucher-Guèvremont, S. \& Chartrand, M. (2019). Actualité de la pauvreté : débats théoriques, défis pratiques. Reflets, 25(1), 10-17.

https://doi.org/10.7202/1064664ar 


\title{
Actualité de la pauvreté : débats théoriques, défis pratiques
}

\author{
Dablia Namian PhD, Professeure agrégée \\ École de service social, Université d'Ottawa \\ Sarah Boucher-Guèvremont, Doctorante \\ École de service social, Université d'Ottawa \\ Martin Chartrand, Doctorant \\ École de service social, Université d'Ottawa
}

La pauvreté fait l'objet, actuellement, d'un intérêt renouvelé en travail social ${ }^{1}$ et dans d'autres sciences sociales. Les plus récentes statistiques montrant l'ampleur du phénomène en Amérique du Nord invitent à s'y intéresser de plus près. Au Canada, selon certaines estimations, ce serait environ 5 million de personnes, soit 1 personne sur 7 , qui vivent sous le seuil de la pauvreté (EDSC, 2016). Si les prestataires de l'aide sociale comptent toujours parmi les plus pauvres au pays, la montée du phénomène des " travailleurs pauvres ", depuis les années 1990, révèle non seulement l'étendue, mais également la transformation des visages de la pauvreté (Johal \& Thirgood, 2016). N'étant plus strictement confinée aux marges sous forme "résiduelle " (telle que conçue durant la période des Trente Glorieuses), la pauvreté interpelle aujourd'hui au Canada un nombre croissant d'individus à risque de vivre, dans la prochaine décennie, une forme d'insécurité au travail (Statistique Canada, 2016).

Bien qu'il existe à l'heure actuelle une pluralité d'indicateurs (MPC, SFR, IPH, etc.) disponibles pour mieux mesurer son ampleur, la pauvreté semble encore principalement définie comme une insuffisance de ressources financières et matérielles pour combler adéquatement les besoins de base. Pourtant, les indicateurs de faible revenu (pauvreté relative et absolue), quoiqu' importants, ne fournissent qu'une image partielle des multiples réalités et facteurs complexes qui définissent le fait d'être pauvre et qui agissent sur le bien-être des personnes. Ainsi, nous pouvons nommer, parmi d'autres : les problèmes de santé et de logement, les barrières d'accès à l'éducation, la moindre participation sociale et civique, le racisme, les discriminations et les préjugés. De plus, ces indicateurs, 
quoiqu'utiles, laissent dans l'ombre la façon dont la pauvreté, au-delà des faibles revenus, s'inscrit dans des rapports sociaux foncièrement inégaux. Pour comprendre et mesurer l'ampleur de la pauvreté, on ne pourrait, en effet, faire l'économie d'une analyse de la croissance des inégalités sociales, tout comme on ne pourrait prétendre se saisir de cette ampleur sans la lier, directement, au démantèlement d'une pluralité de mesures sociales redistributrices de la richesse, depuis les années 1980: éducation publique de qualité et accessible, logements abordables, garderies subventionnées, collectifs et syndicats de travail, etc. Ces mesures, qui sont le fruit de longues luttes historiques menées par une diversité de mouvements sociaux (mouvements féministe, étudiant, syndical, anti-raciste et anti-colonial, etc.), se fragilisent sans cesse, aujourd'hui, au profit d'une société qui érige l'économie comme valeur centrale et indépassable. Comme le mentionne Nadeau : "l'utopie du tout à l'économie dont souffrent nos sociétés en est à imposer la précarité comme l'horizon normal. Elle tente de normaliser la misère du monde dans un jeu de démolition de luttes sociales menées depuis le XIX siècle. » (2016, p. 36)

Si l'avenir des mesures de protection de sociale semblent incertaines face au déclin des idéaux de solidarité et d'égalité qui ont permis leur envol, que doit-on penser, alors, des initiatives actuellement débattues ou expérimentées dans le cadre des plus récents plans de lutte à la pauvreté, telles que le revenu minimum garanti? Ces pratiques permettent-elles de réduire les écarts de richesse ou visent-elles plutôt à répondre aux effets de pauvreté sans agir sur les inégalités sous-jacentes ? Dans la section entrevue de ce numéro, ce sont entre autres à ces questions que répond Dennis Raphael, professeur et chercheur en politiques et en gestion de la santé à l'Université York, dans le cadre d'une entrevue réalisée par Dahlia Namian. Ce dernier y soulève un paradoxe important : si les inégalités de santé sont directement liées aux écarts de revenus et au pouvoir croissant des entreprises, c'est par la santé et la médicalisation des conduites que l'on cherche pourtant à répondre. Autrement dit, plutôt que d'agir à la racine, en s'attaquant aux déterminants sociaux de la santé — en premier lieu, la pauvreté — on responsabilise les personnes pauvres face à leurs " choix de vie ", en faisant d'elles les entrepreneurs de leur santé et de leur bien-être.

\section{Actualité des réponses à la pauvreté : réduction des inégalités ou gestion des symptômes?}

Différent efforts ont été entrepris, au fil des années, en sciences sociales, pour mieux conceptualiser et intervenir sur la pauvreté. En travail social, faut-il le rappeler, la pauvreté fut l'objet d'un débat de fond qui traverse encore aujourd'hui la discipline : 
doit-on " réformer les pauvres " ou agir pour transformer les conditions sociales qui produisent la pauvreté? Au $19^{\mathrm{e}}$ siècle, alors qu'émergent les premiers balbutiements de la discipline professionnelle du travail social, le train de la révolution industrielle bât son plein, enrichissant une minorité de personnes détentrices du capital et propulsant, simultanément au passage, une masse croissante de personnes laissées pour compte, dans l'horizon infernal de la pauvreté. Devant ce spectacle de la misère, les premières travailleuses sociales ont dû centrer leurs efforts pour venir en aide à un nombre critique de personnes et de familles dans le besoin. Or, ce sont sur les modalités et les solutions pour y répondre que certaines tensions idéologiques ont pris forme et se sont cristallisées, parfois de manière paroxystique, autour de deux figures et institutions pionnières du travail social en Amérique du Nord (Garneau et Namian, 2017). D’un côté, dans la lignée de Mary Richmond et la Charity Organisation Society, la primauté devait être accordée au changement individuel; la pauvreté étant principalement perçue comme pouvant être surmontée par la rééducation et la réhabilitation morale des individus et de leur famille (Franklin, 1986). De l'autre côté, dans la lignée cette fois de Jane Addams et du Settlement houses mouvement, l'action directe et collective sur l'environnement quotidien des personnes pauvres (conditions de logement, de travail, etc.) devait être privilégiée pour favoriser un changement social plus global. Il faut dire que ces conceptions divergentes prenaient place dans un contexte où les autorités publiques, face à un paupérisme de masse, étaient soucieuses de gérer plus efficacement l'aide distribuée aux pauvres. Elles souhaitaient à la fois rationnaliser l'assistance publique pour freiner la " dépendance " des personnes pauvres et, en même temps, conjurer la menace grandissante d'une « classe dangereuse ». Dans un climat de soupçon faisant de l'indigent un "profiteur » en puissance (cherchant à multiplier les occasions pour ne pas travailler), le travail social était alors, bien souvent malgré lui, convoité à opérationnaliser un partage entre bons et mauvais pauvres.

Aujourd'hui, alors même que nous entrons dans un nouveau cycle du capitalisme, tourné vers l'automatisation croissante de l'économie sur les lambeaux de son industrialisation, on peut observer, sous de nouveaux habits, un renouveau des tensions idéologiques constitutives du travail social telles qu'elles prirent forme au début du $19^{\mathrm{e}}$ siècle. La croissance actuelle, au Canada, comme dans d'autres sociétés libérales, du nombre de travailleurs et travailleuses pauvres montre la porosité des frontières entre les mondes sociaux autrefois séparés du travail et de l'assistance (Namian \& Binet, 2016) et rend de ce fait caduque, du moins en principe, le partage moral entre bons et mauvais pauvres fondé sur la valeur du travail (Lazarus, 2012). Pourtant, comme le montre le texte de Catherine Charron, de même que celui de Amélie Mauger et Elisabeth Greissler, 
les réformes contemporaines de l'aide sociale renforcent et renouvellent ce partage par l'application des mesures sécuritaires envers les personnes assistées (encore et toujours suspectées de "profiter du système "), ou encore, plus subtilement, par l' "activation " des " capacités " et des ressources personnelles des personnes ciblées comme étant "à risque » de dépendre du système.

Dans un contexte où l'État cherche par divers moyens, subtiles et moins subtiles, à réduire les mesures de protections sociales, à sabrer dans les dépenses et les services publics ainsi qu'à rationaliser toujours davantage l'aide distribuée au nom d'une logique productiviste, force est de constater que le climat de défiance à l'égard des personnes pauvres, qui régnait déjà deux siècles plus tôt, soit malheureusement encore d'actualité. De plus, à ces dynamiques de fond s'ajoute un processus tout autant délétère, prenant racine dans le même foyer néolibéral qui les rendent possibles, soit la judiciarisation croissante de la pauvreté. Comme l'aborde la sociologue et professeure en droit Emmanuelle Bernheim, qui souligne elle aussi un paradoxe fondamental, les recherches démontrent clairement que les personnes les plus judiciarisées vivent dans la précarité, voire dans l'extrême pauvreté. Pourtant, c'est par la judiciarisation que cette pauvreté est traitée et régulée. Qui plus est, le nombre de personnes faisant face à la justice sans avocat ne cesse d'augmenter tandis que l'accès aux services juridiques gratuits ou à faible coût se fait, quant à lui, de plus en plus rare... En somme, ces quatre premiers textes dressent un constat préoccupant sur la manière dont on choisit de répondre, aujourd'hui, à la pauvreté et invite, de ce fait, le travail social, comme d'autres disciplines en sciences sociales, à proposer de nouvelles armes critiques tant pour s'attaquer aux racines idéologiques de la logique néolibérale qu'aux effets qui en découlent pour les personnes touchées.

\section{Pauvreté, intersectionnalité et subjectivité}

Si la pauvreté interpelle aujourd'hui un nombre croissant de personnes, il n'empêche que l'appartenance à certains groupes sociaux, en raison de divers facteurs d'oppressions (le genre, la race, le statut migratoire, l'âge, le handicap mental ou physique, la langue etc.), amplifie les risques d'en subir ses conséquences (Swift $\&$ al., 2010). Tandis que la proportion du seuil de faible revenu est d'environ $8,8 \%$ dans l'ensemble de la population canadienne, des études plus récentes montrent que la catégorie des travailleurs pauvres au Canada est majoritairement composée de personnes racisées et immigrantes (Fleury, 2016; 2007). De plus, les mères monoparentales, les personnes seules et âgées, les personnes autochtones de même que les personnes en situation de handicap sont plus susceptibles 
de vivre en situation de pauvreté et ce à plus long terme (CPJ, 2017). Lorsque combinée ou articulée à d'autres facteurs de minorisation, la langue peut aussi contribuer aux écarts de revenu et au maintien, voire à la reproduction, de la précarité chez les populations francophones minoritaires au Canada, pourtant encore sous-représentées dans la littérature sur la pauvreté au Canada (Molgat \& Trahan-Perreault, 2015; Coderre \& Dubois, 2000; Lacroix \& Nedjar, 1997; Drolet \& al., 2010; Garceau \& Charron, 1997).

Comme l'aborde Jonathan Binet dans son texte en faisant un retour critique sur la rationalité politique sous-jacente à la catégorie des "NEEF », les jeunes constituent de manière générale l'un des groupes sociaux les plus touchés par le chômage, la pauvreté et les effets délétères d'une économie néolibérale qui tend à les propulser dans une trajectoire incertaine et en dents de scie. À cet égard, l'anthropologue Marie-Thérèse Atséna Abogo met de son côté en lumière les effets de l'intrication des facteurs d'oppression de la race et de la classe sur l'environnement et la mobilité sociale des jeunes racisés, issus de l'immigration. À l'aide d'une lecture intersectionnelle et d'une enquête de terrain dans la ville de Québec, elle rend compte de la manière dont le hip-hop, pour ces jeunes, devient à la fois un véhicule d'expression des inégalités et une stratégie de résistance face aux discriminations et obstacles auxquels ils sont confrontés. Le texte de Mélanie Bourque, Katia Grenier, Sylvie Thibault, Maude Champagne et Josée Grenier donne quant à lui la voix à des femmes marginalisées, vivant une situation d'itinérance, en tenant compte de la manière dont les inégalités de genre, la pauvreté, la violence, l'accès aux services et la désinstitutionalisation façonnent leur situation et sont la conséquence d'obstacles liés à l'exercice d'un plein régime de citoyenneté.

Si la pauvreté relève toujours d'un manque (Mercier, 1995), pouvant être saisie comme une "sous-normalité miséreuse ", pour reprendre le terme de Vollmann (2008), soit comme une incapacité à satisfaire un ensemble de besoins socialement créés, l'expression de ce manque est loin de se limiter au revenu ou à la consommation. Les recherches en sciences sociales tentent à cet effet de complexifier et renouveler, depuis une vingtaine d'années, l'analyse strictement économique et positiviste de la pauvreté, en se concentrant sur l'expérience et les conséquences vécues des multiples privations et barrières qu'elle engendre. Or, il reste encore d'importants efforts à faire pour mettre en lumière la «face subjective des inégalités» (Duru-Bellet, 2011), en tenant compte au premier plan de l'expérience et du vécu des personnes pauvres, de même que du stigmate, souvent très sensible, de la pauvreté, qui peut être saisi autant comme une conséquence qu'un facteur aggravant (Lamont \& Mizrachi, 2012). Comment les personnes vivent et font face aux stigmates associés à la pauvreté ? Comment perçoivent-elles la pauvreté et les inégalités qui 
marquent leur trajectoire? Quels sentiments éprouvent-elles du fait d'être catégorisées ou stigmatisées comme pauvres? C'est pour répondre en partie à ces questions que le texte de Isabel Lanteigne, Lise Savoie, Hélène Albert et Catherine Roy-Comeau, à partir des récits de vie collectés dans le cadre de trois recherches qualitatives au Nouveau Brunswick, place la focale sur la souffrance des femmes en situation de pauvreté. Leur article met en lumière trois dimensions de cette souffrance, soit la disqualification, la stigmatisation et les modes d'adaptation et de résistance. Dans cette veine, François Xavier Charlebois présente, quant à lui, les épreuves sociales vécues par dix jeunes adultes en situation de pauvreté et de raccrochage scolaire. Son analyse, à partir d'histoires de vie, permet une compréhension complexe de leur processus de construction identitaire en identifiant les contraintes persistantes et les possibilités de leur émancipation.

Pour compléter ce dossier, la section Pratique à notre image présente un récit de pratique écrit par Martin Chartrand et Yves Séguin, au sujet d'une équipe mobile en itinérance en Outaouais. Ce récit de pratique fait état des complexités associées au fait d'intervenir auprès des personnes sans-abri qui sont fortement précarisées et isolées, de même que des enjeux associés à l'approche SRA (Stabilité résidentielle avec accompagnement) privilégiée par le gouvernement fédéral. Enfin, pour poursuivre la réflexion sur les solutions proposées pour répondre à la pauvreté, la section $L u$ pour vous présente l'ouvrage Utopies réalistes de Rudger Bregman (2017), recensé par Sara Lambert.

\section{Bibliographie}

CITIZENS FOR PUBLIC JUSTICE (CPJ) (2017). Poverty Trends Report 2017, Réf du $1^{\text {er }}$ octobre 2018, https:/www.cpj.ca/sites/default/files/docs/files/PovertyTrendsReport2017.pdf

CODERRE, Cécile, et Madeleine DUBOIS (2000). "Solidarité et citoyenneté : initiatives pour contrer la pauvreté chez les francophones dans Ottawa-Carleton ", Reflets : revue d'intervention sociale et communautaire, Vol. 6, No 2, p. 61-86.

DROLET, Marie, Stéphanie GARNEAU ET Madeleine DUBOIS (2010). "L'intervention sociale en contextes minoritaires : penser la complexité et la multiplicité des processus de minorisation ". Reflets : revue d'intervention sociale et communautaire, Vol. 16, No. 2, p. 10- 19, DOI:10.7202/1000312ar

DURU-BELLET, M. (2011). « La face subjective des inégalités. Une convergence entre psychologie sociale et sociologie? ", Sociologie, $\mathrm{N}^{\circ} 2$, vol. 2 [en ligne] 
EMPLOI ET DÉVELOPPEMENT SOCIAL CANADA (EDSC) (2016), Document d'information sur la pauvreté au Canada, Gouvernement du Canada, Réf du 7 février 2018,

https://www.canada.ca/fr/emploi-developpement-social/programmes/reduction-pauvrete/ document-information.html

FLEURY, Dominique (2007). A Study of Poverty and Working Poverty Among Recent Immigrants to Canada, Ottawa, Human Resources and Social Development Canada, 64 p.

FLEURY, Dominique (2016). Precarious Employment in Canada: An Overview of the Situation, Hill Notes, Library of Parliament, Réf. 7 février 2018,

https://hillnotes.ca/2016/01/27/precarious-employment-in-canada-an-overview-of-the-situation/

FRANKLIN, Donna L. (1986). "Mary Richmond and Jane Addams : From Moral Certainty to Rational Inquiry in Social Work Practice ", Social Service Review, Vol. 60, No. 4, p. 504-525

GARCEAU, Marie-Luce et Marc CHARRON (1997). «La dynamique de la pauvreté : l'exemple des Franco-Ontariennes de 45 à 64 ans », Revue du Nouvel-Ontario, Vol. 19, p. 39-58.

GARNEAU, Stéphanie et Dahlia NAMIAN (2017). Goffman et le travail social, Ottawa, Presses de l'Université d'Ottawa, $320 \mathrm{p}$.

LACROIX, Colette B. et NEDJAR Yasmine (1997). "Alphabétisation et lutte contre la pauvreté chez les francophones d'Ottawa-Carleton ", Reflets : revue d'intervention sociale et communautaire, Vol. 3, No 1, p. 153-157.

LAMONT, Michèle et Nassim MIZRACHI (2012). Responses to Stigmatization in Comparative Perspective, New York, Routledge, 188 p.

LAZARUS, Jeanne (2012). «Les enjeux de la sociologie de la pauvreté ». Ceriscope Pauvreté, Réf du 15 février 2018, http://ceriscope.sciences-po.fr/pauvrete/content/part1/les-enjeux-de-la-sociologiede-la-pauvrete?page $=1$

MERCIER, Lucie (1995). «La pauvreté : phénomène complexe et multidimensionnel ». Service social, Vol. 44, n³, p. 7-27.

MOLGAT, Marc et Julienne TRAHAN-PERREAULT (2015) "L'état de la recherche en travail social dans les communautés francophones minoritaires au Canada : une topographie thématique ", Reflets : revue d'intervention sociale et communautaire, Vol. 21, n 2, p. 36-70, DOI:10.7202/1035433ar

NADEAU, Jean-François (2016). Les radicaux libres. Montréal, Lux Éditeur, 322p.

NAMIAN, Dahlia et Jonathan BINET (2016). « Politiques d'activation et nouvelles « frontières » du travail social ", dans Lilian NEGURA (dir.). L'intervention en sciences humaines : l'importance des représentations, Québec, Presses de l'Université Laval, p. 99-128. 
STATISTIQUE CANADA (2016). Tableau 1: Caractérstiques de la population activie selon l'âge et le sexe-Données désaisonnalisées, réf. du 19 mars 2018, http://www150.statcan.gc.ca/n1/daily-quotidien/161104/t001a-fra.htm

SWIFT, Jamie, Brice BALMER et Mira DINEEN (2010). Persistent poverty: Voices from the margins, Toronto, Between the Lines, 184 pages.

THIRGOOD, Jordann et Sunil JOHAL (2016). Working Without a Net: Rethinking Canada's social policy in the new age of work, Mowat Center, University of Toronto, 60 pages.

VOLLMANN, William T. (2008). Pourquoi êtes-vous pauvres? Paris, Actes Sud, 416p.

\section{Note}

1 En témoigne notamment le dernier appel à contribution de la revue de l'Association Canadienne pour la formation en travail social (ACFTS) 